\title{
A rare case of ectopic ACTH syndrome caused by primary renal neuroendocrine tumor
}

\author{
Paweena Chunharojrith ${ }^{1}$, Kanapon Pradniwat ${ }^{2}$ and Tanawan Kongmalai(i)1 \\ 1Division of Endocrinology and Metabolism, Department of Medicine and 2Department of Pathology, Faculty of \\ Medicine Siriraj Hospital, Mahidol University, Bangkok, Thailand
}

Correspondence should be addressed to T Kongmalai Email

Tanawan.api@mahidol.ac.th

\section{Summary}

Ectopic adrenocorticotropic hormone (ACTH) secretion is responsible for 5-15\% of Cushing's syndrome (CS). Neuroendocrine tumor (NET) is a common cause of ectopic ACTH syndrome (EAS). However, primary renal NET is exceedingly rare. Fewer than 100 cases have been reported and only a few cases presented with CS. Because of its rarity and lack of long-term follow-up data, clinical manifestations, biological behavior and prognosis are not well understood. Here, we report the case of a 51-year-old man who presented with clinical and laboratory findings compatible with EAS. CT scan revealed a lesion of uncertain nature at the lower pole of the left kidney. Octreotide scan found a filling defect at the lower pole of left kidney. It was difficult to determine if this finding was the true etiology or an incidental finding. Unfortunately, the patient's clinical status rapidly deteriorated with limited medical treatment. The patient underwent left nephrectomy and left adrenalectomy. Histopathological examination confirmed NET with oncocytic features. Immunohistochemistry staining was positive for ACTH. The patient's condition gradually improved. Additionally, glucocorticoid replacement was required only 6 months during a gradual recovery of hypothalamic pituitary adrenal axis achieved approximately three years after tumor removal. Although extremely rare, primary renal NET should be considered as a cause of EAS particularly in a patient with rapid clinical deterioration. Thorough investigation, early diagnosis and careful management are crucial to reduce morbidity and mortality.

\section{Learning points:}

- Primary renal NET is an extremely rare cause of ectopic ACTH syndrome.

- Ectopic ACTH syndrome has a rapid onset with severe clinical manifestations. In this case, the patient's condition deteriorated rapidly, resulting from severe hypercortisolism. Resection of the tumor is the most effective treatment.

- Localization of ectopic ACTH-secreting tumors is very challenging. Multimodality imaging including CT, MRI, octreotide scan, and positron emission tomography plays a crucial role in identifying the tumors. However, each imaging modality has limitations.

\section{Background}

Cushing syndrome (CS) comprises signs and symptoms of chronic exposure to hypercortisolism leading to metabolic, infectious and cardiovascular complications. Ectopic adrenocorticotropic hormone secretion (EAS) is responsible for $5-15 \%$ of all CS cases $(1,2)$. High adrenocorticotropic hormone (ACTH) secretion and the malignant behavior of this tumor are associated with rapid clinical deterioration and up to a 13.3-fold increase in mortality (3). Hence, early diagnosis and proper management are essential. The major of causes of EAS include small cell lung carcinoma, bronchial carcinoid, pancreatic tumor, thymic carcinoid, medullary thyroid 
cancer and non-small cell lung carcinoma (4). Primary renal neuroendocrine tumor (NET) is an exceedingly rare cause of EAS. According to our extensive literature review, fewer than 100 cases of primary renal NET have been reported in the English literature $(5,6)$. Interestingly, only two cases presented with CS $(7,8)$. Here we report the third case of primary renal NET presenting with EAS complicated by diabetes mellitus, active pulmonary tuberculosis, seizures and hepatitis that posed challenges in localization and management.

\section{Case presentation}

A 51-year-old man was referred to our hospital due to suspicion of CS. He presented with one month of hypokalemic metabolic alkalosis (potassium $2.8 \mathrm{mmol} / \mathrm{L}$, HCO3 $35 \mathrm{mmol} / \mathrm{L}$ ), pitting edema of both legs, and diabetes mellitus. Otherwise, his medical history was unremarkable. Initial physical exam revealed BMI was 21.6 $\mathrm{kg} / \mathrm{m}^{2}$ and blood pressure was $169 / 105 \mathrm{mmHg}$ without any sign of long-standing hypertension. Other physical parameters were normal. No cushingoid appearance or hyperpigmentation was found.

\section{Investigation}

Biochemical assessment confirmed the diagnosis of endogenous CS with very high ACTH levels (Table 1). To differentiate between Cushing's disease and EAS, highdose dexamethasone suppression test was performed and the result was non-suppressible. A pituitary MRI demonstrated an empty sella; no mass was identified. As a result, bilateral inferior petrosal sinus sampling (BIPSS) was planned to differentiate between a small ACTHsecreting pituitary adenoma which may be undetected by MRI and EAS.
In the meantime, CT of neck, thorax and abdomen were performed to localize the source of ACTH production. CT of the whole abdomen showed a $2.8 \times 2.7$ $\mathrm{cm}$ well-defined, slightly hyperdense lesion $(44 \mathrm{HU})$ at the lower pole of the left kidney with mild enhancement on 10-minute delay phase (79 $\mathrm{HU})$. There was no associated mural nodule or adjacent perinephric fat stranding suspicious of hemorrhagic cyst or hypovascular subtypes of renal cell carcinoma (RCC) such as papillary RCC (Fig. 1).

Since the nature of the left renal lesion was uncertain and RCC has been found to account for only $2 \%$ of all neoplasms that are responsible for CS (9). This finding was possibly unrelated to the disease or was the true etiology of ACTH production. Thus, whole body venous sampling (BIPSS, bilateral femoral vein, superior vena cava, inferior vena cava and renal veins) was scheduled. Unfortunately, the patient developed multiple drug-resistant pulmonary tuberculosis (MDR-TB). Anti-tuberculosis drugs were initiated and all procedures had to be postponed for at least two weeks.

\section{Treatment}

In light of the very high cortisol levels and active MDR-TB infection, rapid pharmaceutical control of hypercortisolism was our immediate objective. However, mitotane and mifepristone are not available in Thailand. Ketoconazole was not applicable because concomitant use with anti-tuberculosis drugs would enhance the risk of hepatitis. Therefore, pasireotide was initiated. One week later, the patient was admitted to a nearby hospital due to an unprovoked seizure. Blood examination, lumbar puncture and brain CT were normal. Phenytoin was prescribed for seizure control.

Table 1 Results of laboratory investigations and reference values.

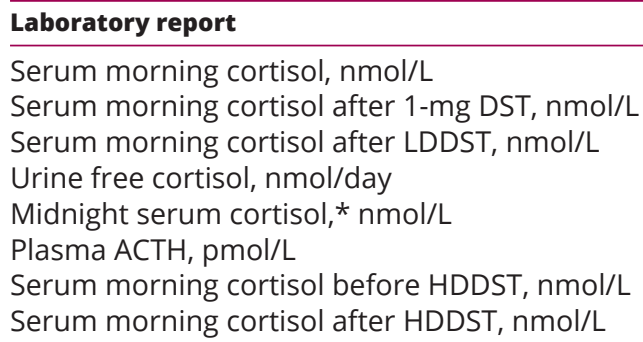

\begin{tabular}{c}
\hline Result \\
\hline 2002.8 \\
1702.1 \\
1624.8 \\
$>5009.9,3757.5$ \\
1906.2 \\
$489.3,542.4$ \\
1624.8 \\
1566.9
\end{tabular}

\begin{tabular}{c}
\hline Normal value \\
\hline $165.5-507.6$ \\
$<49.7$ \\
$<49.7$ \\
$0-413.8$ \\
$<49.7$ \\
$2.2-13.2$
\end{tabular}

*During sleep.

1-mg DST, overnight 1 mg dexamethasone suppression test; ACTH, Adrenocorticotropic hormone; HDDST, high dose dexamethasone suppression test; LDDST, low dose dexamethasone suppression test. 


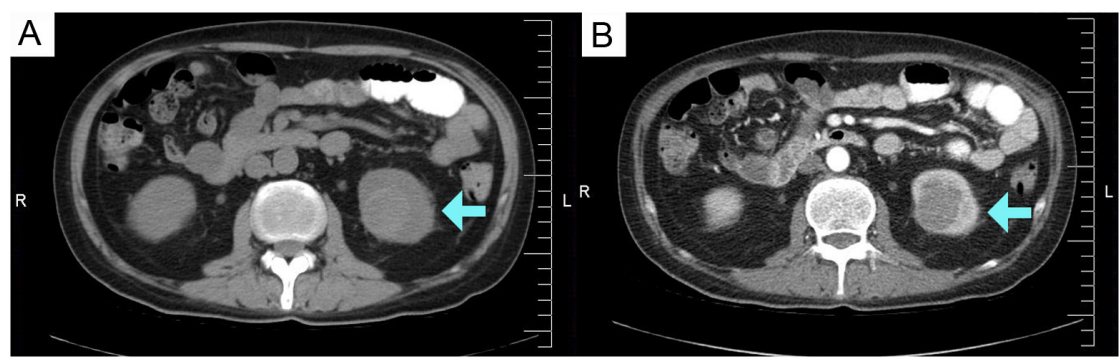

\section{Figure 1}

CT imaging axial view, without (A) and with (B) IV contrast, showed a $2.8 \times 2.7 \mathrm{~cm}$ well-defined, slightly hyperdense lesion ( $44 \mathrm{HU}$ ) at the lower pole of the left kidney with mild enhancement on arterial phase (blue arrow), suspicious of hemorrhagic cyst or hypovascular subtypes of renal cell carcinoma.
Two weeks later, he was admitted to our hospital due to phenytoin and anti-tuberculosis drugs induced hepatitis. Despite the lack of efficacy data on pasireotide therapy in EAS, the patient showed positive biochemical response to pasireotide (serum morning cortisol: $653.8 \mathrm{nmol} / \mathrm{L}$, urine free cortisol (UFC): $1495.4 \mathrm{nmol} /$ day and ACTH: 225.9 $\mathrm{pmol} / \mathrm{L})$. However, the medication had to be discontinued due to hepatitis. The patient's clinical condition rapidly declined which limited medical treatment options. Surgical removal of the tumor was determined to be the most effective therapy. To address our concern that the left renal lesion might not be the true source of ectopic ACTH production, a somatostatin receptor scintigraphy (SRS) with Tc-99m HYNIC-TOC was performed. The scan identified an increased abnormal radioactivity accumulation in pulmonary infiltration of the right upper lung compatible with active pulmonary tuberculosis and no evidence of a somatostatin receptor positive tumor in the left kidney. Due to his poor medical condition, surgery represented a very high risk of perioperative morbidity and mortality. After extensive discussion with the medical teams and his family, the patient underwent urgent left radical nephrectomy and left adrenalectomy. A $3.5 \mathrm{~cm}$ well-circumscribed dark red hemorrhagic renal mass was found at the lower pole. Histopathological examination revealed a NET with oncocytic features and extensive necrosis in the hemorrhagic background rimmed by neutrophilic infiltration, likely representing infarction. Minimal viable tumor was seen at the periphery imparting morphology reminiscent of well-differentiated NET with oncocytic changes. Mitoses were present up to 2/10 HPFs in rare viable areas. No lymphovascular invasion was identified. Immunohistochemistry was positive for ACTH staining (Fig. 2).

\section{Outcome and follow-up}

Four days after an uneventful operation, serum morning cortisol was $85.5 \mathrm{nmol} / \mathrm{L}$, ACTH was $116.2 \mathrm{pmol} / \mathrm{L}$. Adrenal insufficiency was diagnosed and glucocorticoid replacement therapy was prescribed. UFC was normal

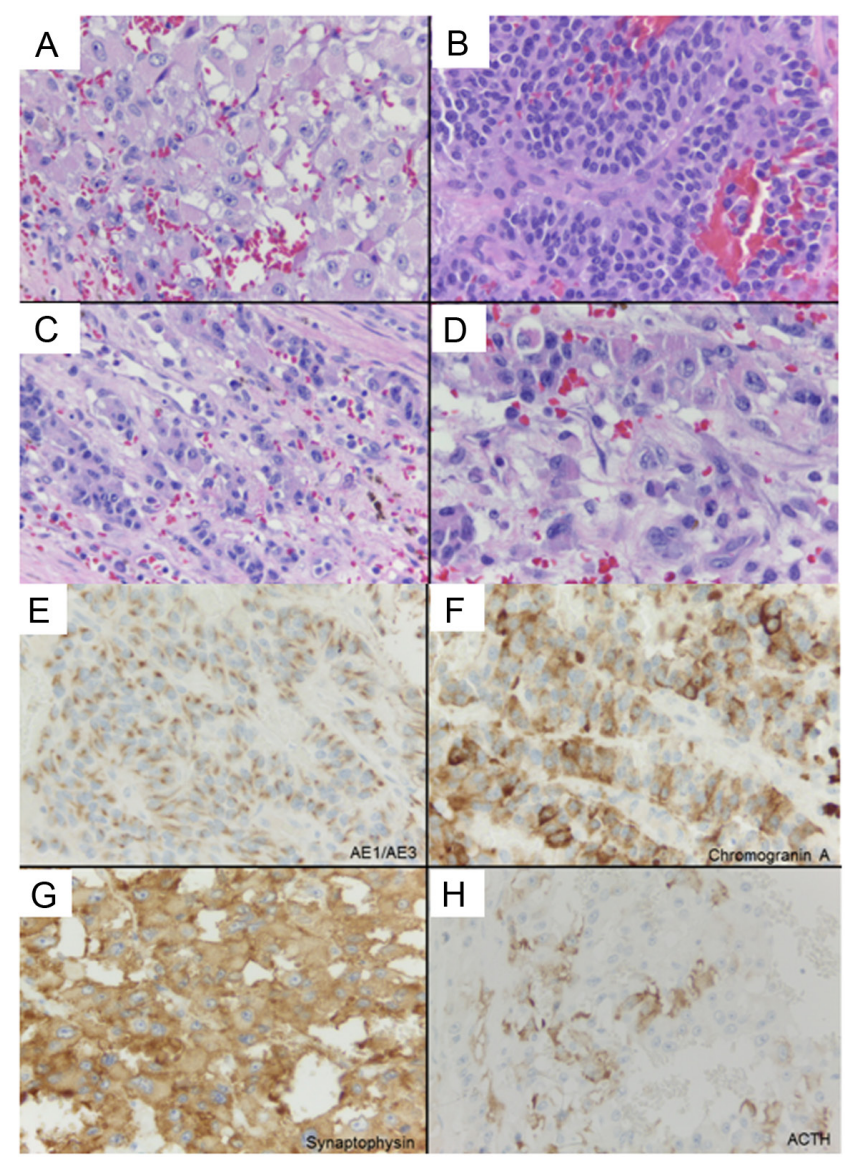

Figure 2

Histopathological findings and immunohistochemical studies of the tumor. (A) Majority of viable tumor cells showed oncocytic cell change with abundant cytoplasm and conspicuous nucleoli, potentially mimicking renal cell carcinoma. (B) Classical features of neuroendocrine tumor were focally present imparting relatively high N/C ratio and speckled chromatin. (C) Oncocytic cellular feature with uniform nuclei and some amount of granular eosinophilic cytoplasm resembling renal oncocytoma. (D) Oncocytic cell changes depicted bulk of eosinophilic granular cytoplasm. Note the expansion of cytoplasmic border which push nuclei aside, more or less resembling rhabdoid cells. The finding should not be misinterpreted as high-grade renal cell carcinoma harboring rhabdoid cell feature. (E) AE1/AE3 with paranuclear dot staining pattern. $(F)$ and $(G)$ Cytoplasmic staining with neuroendocrine markers, chromogranin and synaptophysin. (H) Focal cytoplasmic staining with ACTH immunohistochemistry. Focal CK7 positivity was also observed. PAX8, vimentin, S100, CD117, AMACR, HMB45, and Melan A were negative. 


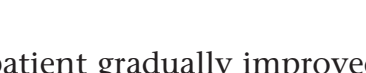

one month after surgery. The patient gradually improved and completed the MDR-TB treatment regimen. Morning serum cortisol level increased slowly up to above 275.9 $\mathrm{nmol} / \mathrm{L}$ within 6 months after tumor resection. Therefore, glucocorticoid replacement was withdrawn without the clinical manifestations of adrenal insufficiency. Notably, the serum ACTH remained elevated (range from 26 to $99.3 \mathrm{pmol} / \mathrm{L}$ ), raising concerns for recurrent or metastatic disease. Morning serum cortisol and ACTH after $1 \mathrm{mg}$ overnight dexamethasone test was $8.3 \mathrm{nmol} / \mathrm{L}$ and 2.8 pmol/L, respectively (Fig. 3). Abdominal CT found no evidence of tumor recurrence and the size of the right adrenal gland decreased. Moreover, three years after surgery the UFC remained within the normal range. Sequential cosyntropin stimulation tests were performed at 18 months after tumor removal. Peak serum cortisol response after $250 \mu \mathrm{g}$ ACTH stimulation test was 369.7 $\mathrm{nmol} / \mathrm{L}$, suggesting that the hypothalamic-pituitaryadrenal (HPA) axis had not fully recovered. At three years of follow-up morning serum cortisol and ACTH levels were $408.3 \mathrm{nmol} / \mathrm{L}$ and $15.8 \mathrm{pmol} / \mathrm{L}$, respectively. Patient discontinued antidiabetes and antihypertensive medications and he was healthy.

\section{Discussion}

With extremely high plasma ACTH and cortisol levels, EAS develops rapidly with severe clinical deterioration. The delay in identifying the source of ACTH production and in reducing cortisol level can cause significant morbidity and mortality. EAS is a diagnostic challenge because most cases do not present typical cushingoid features. Most patients present with sudden onset of hypertension, hypokalemia and edema of both lower limbs, as found in our patient $(4,10)$.

The most commonly reported causes of EAS are small cell lung cancer and NET (1). NETs are characteristically low-grade neoplasms arising from specific neuroendocrine cells which are capable of amine precursor uptake and decarboxylation. The majority of NETs are found in the gastrointestinal (66.9\%) and respiratory tract $(24.5 \%)$ (11). For the reason that enterochromaffin cells are not found in normal kidney, NETs are unusual found in adult renal parenchyma, pelvis or ureters. Hence, primary renal NET is exceedingly rare. In 1966 Resnick et al. first described these tumors (12) and since that time less than 100 cases have been reported in the English literature. Due to its rarity, the pathogenesis is still debated (13). Some experts believe that these tumors arise from interspersed neuroendocrine cells associated with acquired and/or congenital abnormalities (14), while others believe that certain renal cells may undergo intestinal metaplasia and give rise to NET $(15,16)$.

The mechanism for ectopic ACTH production in the tumor is not well established. One prevailing hypothesis is that ACTH production results from aberrant transcription and overexpression of proopiomelanocortin (POMC) in the tumor. POMC cleavage by prohormone convertase PC $1 / 3$ gives rise to ACTH. In normal non-pituitary tissue, the POMC gene is transcripted by the P3 promoter causing shorter mRNA that cannot be translated. Nevertheless, P2 or P1 promoter is used to generate a pituitary size mRNA in ACTH-secreting tumors. Therefore, EAS represents a cancer-induced escape from genomic controls that exists in normal tissue $(17,18)$.

Primary renal NET usually presents with abdominal, back or flank pain. In almost one-third of cases, the tumor is discovered incidentally or during routine check-up, rarely presenting with CS (19). In 1988 Hannah et al. first described a case of a 12.8-year-old girl who presented with three severe episodes of active CS caused by NET of the left kidney. Subsequently, in 2019, Kocher et al. reported a case of an 11-year-old girl presenting with cushingoid features and a left-sided flank mass. The final pathology

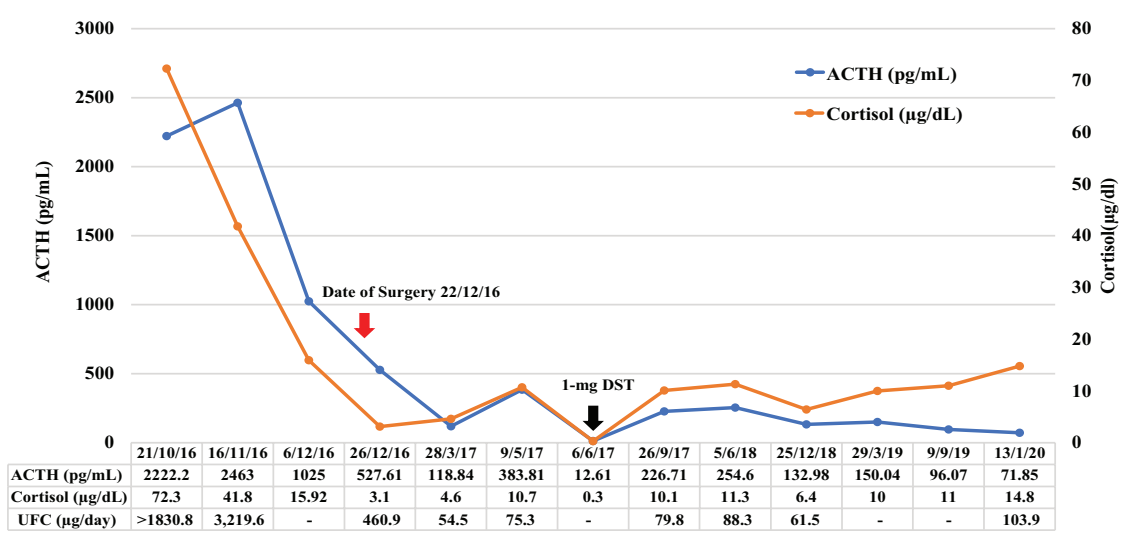

\section{Figure 3}

Circulating plasma ACTH and serum cortisol since the presentation and follow up after surgery (1-mg DST, overnight $1 \mathrm{mg}$ dexamethasone suppression test; ACTH, Adrenocorticotropic hormone; UFC, Urine free cortisol). Conversion factors for units: plasma ACTH from $\mathrm{pg} / \mathrm{mL}$ to $\mathrm{pmol} / \mathrm{L}, 0.22$; serum cortisol from $\mu \mathrm{g} / \mathrm{dL}$ to $\mathrm{nmol} / \mathrm{L}, 27.59$; urine free cortisol from $\mu \mathrm{g} /$ day to $\mathrm{nmol} / \mathrm{day}, 2.759$. 
confirmed a high-grade NET with nodal metastasis (8). Differently from the above-mentioned article, our patient is the first reported case in an adult. He developed CS at 51 years of age with extensive tumor necrosis on histological study. However, similarly to the first case report, oncocytic changes that are not commonly seen in NET were found in both cases.

The differential diagnosis of the left renal lesion included RCC, which is the most common tumor arising from the kidney, and NET. Only $2 \%$ of CS is caused by RCC and renal NET has no specific radiologic feature on CT or MRI. Thus, imaging alone is unreliable to differentiate between these two diseases (20). SRS has been introduced as a sensitive imaging for diagnosis and staging of NET. Although it has a high sensitivity in detecting gastrointestinal and bronchial tumors (85\%), little is known about the sensitivity and prevalence of somatostatin receptors in renal NET. Moreover, the normal kidney uptake of a tracer material may obscure a suspicious lesion (21). In our patient, SRS demonstrated a filling defect at the lower pole of the left kidney. This may have been due to an extensive necrosis found on pathological examination, the presence of unlabeled somatostatin on the tumor that lowered its detectability. Another possibility is that the active tuberculosis infection may have absorbed the tracer material resulting in negative findings at the kidney. Additionally, hypercortisolism can suppress tumoral somatostatin subtype 2 receptor concentrations, potentially causing false-negative results (2). While 18F-FDG PET has been reported to offer little additional diagnostic value, ${ }^{68} \mathrm{Ga}$-DOTATATE PET/CT has demonstrated promising results for detection of NET (sensitivity 90\%, specificity $80 \%$ ) (22). However, this novel functional imaging is not available in our institute.

The histological findings found variant cytomorphologic features and extensive tumor degeneration that prevented complete morphologic assessment. Classical carcinoid tumor features that allow recognition of NET were scant and only demonstrated by thorough tumor sampling. Oncocytic cell changes in this tumor could create a potential diagnostic pitfall, mainly renal oncocytoma and RCC depending on areas evaluated. Despite its close resemblance to renal oncocytoma, the presence of conspicuous nucleoli may be a clue against oncocytoma. Distinguishing between NET and RCC can be difficult based on morphology alone. A complete immunohistochemistry (IHC) panel is useful. RCC, with the exception of histologic grade 4 RCC, is almost always positive for PAX8 while NET is not. Renal oncocytoma is frequently positive for CD117 and negative for chromogranin and synaptophysin. IHC staining in our patient was positive for chromogranin and synaptophysin which was compatible with NET. Moreover, IHC revealed cytoplasmic staining with ACTH that confirmed the source of ectopic ACTH production.

Generally, the median time to HPA axis recovery in EAS after successful surgery was 0.6 years and the probability of recovery at 5 years in EAS was 82\% (23). In our patient, adrenocortical function recovery after surgery was rapid and glucocorticoid was discontinued within 6 months. However, it had not fully recovered. Over a period of a 3 -year of follow-up, morning serum cortisol increased gradually suggesting that the HPA axis had gradually recovered. According to the short duration and the paucity of the cases, clinical manifestations and histopathological behavior are not well understood. Moreover, lack of longterm follow-up data leaves considerable uncertainty in the prognosis.

In conclusion, ectopic ACTH syndrome is a rare and life-threatening condition. Diagnosis, localization and management are challenging. Although extremely rare, primary renal neuroendocrine tumor should be considered as a cause of ectopic ACTH production particularly in a patient with rapid clinical deterioration. Thorough investigation, early diagnosis, and careful management are crucial to reduce morbidity and mortality.

\section{Declaration of interest}

The authors declare that there is no conflict of interest that could be perceived as prejudicing the impartiality of the research reported.

\section{Funding}

This research did not receive any specific grant from any funding agency in the public, commercial or not-for-profit sector.

\section{Patient consent}

Written informed consent has been obtained from the patient for the publication of all data and images of this case report.

\section{Author contribution statement}

$\mathrm{P}$ Chunharojrith and $\mathrm{T}$ Kongmalai were involved in the diagnosis and management of the case. K Pradniwat provided pathological diagnosis. $\mathrm{P}$ Chunharojrith and T Kongmalai wrote and reviewed the manuscript.

\section{References}

1 Isidori AM, Kaltsas GA, Pozza C, Frajese V, Newell-Price J, Reznek RH, Jenkins PJ, Monson JP, Grossman AB \& Besser GM. 
The ectopic adrenocorticotropin syndrome: clinical features, diagnosis, management, and long-term follow-up. Journal of Clinical Endocrinology and Metabolism 200691 371-377. (https://doi. org/10.1210/jc.2005-1542)

2 Lacroix A, Feelders RA, Stratakis CA \& Nieman LK. Cushing's syndrome. Lancet 2015386 913-927. (https://doi.org/10.1016/ S0140-6736(14)61375-1)

3 Yaneva M, Kalinov K \& Zacharieva S. Mortality in Cushing's syndrome: data from 386 patients from a single tertiary referral center. European Journal of Endocrinology 2013169 621-627. (https:// doi.org/10.1530/EJE-13-0320)

4 Isidori AM \& Lenzi A. Ectopic ACTH syndrome. Arquivos Brasileiros de Endocrinologia e Metabologia 200751 1217-1225. (https://doi. org/10.1590/s0004-27302007000800007)

5 Korkmaz T, Seber S, Yavuzer D, Gumus M \& Turhal NS. Primary renal carcinoid: treatment and prognosis. Critical Reviews in Oncology/Hematology 201387 256-264. (https://doi.org/10.1016/j. critrevonc.2013.02.003)

6 Kloppel G. Tumour biology and histopathology of neuroendocrine tumours. Best Practice and Research: Clinical Endocrinology and Metabolism 200721 15-31. (https://doi.org/10.1016/j. beem.2007.01.004)

7 Hannah J, Lippe B, Lai-Goldman M \& Bhuta S. Oncocytic carcinoid of the kidney associated with periodic Cushing's syndrome. Cancer 198861 2136-2140. (https://doi.org/10.1002/10970142(19880515)61:10<2136::aid-cncr2820611034>3.0.co;2-p)

8 Kocher NJ, Loloi J, Warrick J, Burns AS \& Decter RM. Ectopic ACTHsecreting neuroendocrine tumor: a rare etiology of a pediatric solid renal mass. Canadian Journal of Urology 201926 9956-9959.

9 Palapattu GS, Kristo B \& Rajfer J. Paraneoplastic syndromes in urologic malignancy: the many faces of renal cell carcinoma. Reviews in Urology 20024 163-170.

10 Salgado LR, Fragoso MC, Knoepfelmacher M, Machado MC, Domenice S, Pereira MA \& de Mendonça BB. Ectopic ACTH syndrome: our experience with 25 cases. European Journal of Endocrinology 2006155 725-733. (https://doi.org/10.1530/ eje.1.02278)

11 Modlin IM, Lye KD \& Kidd M. A 5-decade analysis of 13,715 carcinoid tumors. Cancer 200397 934-959. (https://doi.org/10.1002/ cncr.11105)

12 Resnick ME, Unterberger H \& McLoughlin PT. Renal carcinoid producing the carcinoid syndrome. Medical Times 196694 895-896.
13 Li B, Cui T, Ban Z, Luo L \& Sun L. Primary renal carcinoid tumor: case report and review of the literature. OncoTargets and Therapy 2016 9 741-743. (https://doi.org/10.2147/OTT.S88730)

14 Shurtleff BT, Shvarts O \& Rajfer J. Carcinoid tumor of the kidney: case report and review of the literature. Reviews in Urology 20057 229-233.

15 Gordon A. Intestinal metaplasia of the urinary tract epithelium. Journal of Pathology and Bacteriology 196385 441-444. (https://doi. org/10.1002/path.1700850224)

16 Yoo J, Park S, Jung Lee H, Jin Kang S \& Kee Kim B. Primary carcinoid tumor arising in a mature teratoma of the kidney: a case report and review of the literature. Archives of Pathology and Laboratory Medicine 2002126 979-981. (https://doi.org/10.1043/00039985(2002)126<0979:PCTAIA>2.0.CO;2)

17 White A \& Clark AJ. The cellular and molecular basis of the ectopic ACTH syndrome. Clinical Endocrinology 199339 131-141. (https:// doi.org/10.1111/j.1365-2265.1993.tb01765.x)

18 Terzolo M, Reimondo G, Ali A, Bovio S, Daffara F, Paccotti P \& Angeli A. Ectopic ACTH syndrome: molecular bases and clinical heterogeneity. Annals of Oncology 200112 S83-S87. (https://doi. org/10.1093/annonc/12.suppl_2.s83)

19 Romero FR, Rais-Bahrami S, Permpongkosol S, Fine SW, Kohanim S \& Jarrett TW. Primary carcinoid tumors of the kidney. Journal of Urology 2006176 2359-2366. (https://doi.org/10.1016/j.juro.2006.07.129)

20 Lamb L \& Shaban W. Primary renal carcinoid tumor: a radiologic review. Radiology Case Reports 20149 923. (https://doi.org/10.2484/ rcr.v9i2.923)

21 Mufarrij P, Varkarakis IM, Studeman KD \& Jarrett TW. Primary renal carcinoid tumor with liver metastases detected with somatostatin receptor imaging. Urology 200565 1002. (https://doi.org/10.1016/j. urology.2004.11.042)

22 Fallahi B, Manafi-Farid R, Eftekhari M, Fard-Esfahani A Emami-Ardekani A, Geramifar P, et al. Diagnostic efficiency of 68Ga-DOTATATE PET/CT as compared to 99mTc-octreotide SPECT/ CT and conventional morphologic modalities in neuroendocrine tumors. Asia Oceania Journal of Nuclear Medicine and Biology 20197 129-140. (https://doi.org/10.22038/AOJNMB.2019.39392.1263)

23 Berr CM, Di Dalmazi G, Osswald A, Ritzel K, Bidlingmaier M, Geyer LL, Treitl M, Hallfeldt K, Rachinger W, Reisch N, et al. Time to recovery of adrenal function after curative surgery for Cushings syndrome depends on etiology. Journal of Clinical Endocrinology and Metabolism 2015100 1300-1308. (https://doi.org/10.1210/jc.2014-3632)

Received in final form 13 February 2021

Accepted 11 March 2021 